\title{
Eine Analytik der Biopolitik. Überlegungen zu Geschichte und Gegen- wart eines umstrittenen Begriffs
}

\author{
Thomas Lemke
}

\begin{abstract}
Der Begriff der Biopolitik hat in den letzten Jahren eine erstaunliche Resonanz gefunden, wobei sich heterogene, ja konträre Verwendungsweisen beobachten lassen. Der Beitrag macht einen Systematisierungsvorschlag, der Klarheit in das begriffliche Wirrwarr bringen soll.

Ausgangspunkt für das hier vorgeschlagene Analyseraster ist die polare Grundkonstellation, die in der Zusammenführung von Leben und Politik zum Begriff der Biopolitik prinzipiell angelegt ist. Entsprechend lassen sich naturalistische Konzepte, die das Leben als vorpolitische Grundlage der Politik begreifen, von politizistischen Ansätzen abgrenzen, die Lebensprozesse als außerpolitischen Gegenstand der Politik in den Blick nehmen. Die historischen Entstehungskontexte und die analytischen Varianten der zwei Interpretationslinien werden in den ersten beiden Teilen des Beitrags vorgestellt. Die Hauptthese ist, dass beide Ansätze zentrale Dimensionen biopolitischer Prozesse nicht erfassen. Gegenüber der naturalistischen und der politizistischen Lesart soll im dritten Teil ein relationaler Begriff der Biopolitik profiliert werden, den als Erster der französische Philosoph und Historiker Michel Foucault entwickelt hat. Die in der Rezeption des foucaultschen Begriffs der Biopolitik formulierten Korrekturvorschläge und die theoretischen Weiterentwicklungen lassen sich in einer Analytik der Biopolitik synthetisieren, die im vierten Teil des Beitrags präsentiert und bioethischen Reflexions- und Analyseformen gegenübergestellt wird.
\end{abstract}

Keywords: Biopolitik; Foucault; politische Theorie; Leben; Biotechnologien

Der Begriff der Biopolitik hat eine bemerkenswerte Karriere hinter sich. ${ }^{1}$ War er bis vor kurzem nur wenigen Fachleuten bekannt, findet er heute eine immer größere Resonanz. Das Spektrum seiner Verwendungsweisen reicht inzwischen von der Asyl-Politik über die AIDS-Prävention bis hin zu Fragen des Bevölkerungswachstums. Er bezeichnet die Förderung landwirtschaftlicher Produkte ebenso wie die Regulierung medizinischer Forschung, strafrechtliche Bestimmungen zur Abtreibung und Patientenverfügungen zum Lebensende. ${ }^{2}$ Aber nicht nur die Gegenstände, auch die Wertungen fallen weit auseinander. Bei „Biopolitik“ denken die einen an eine rationale und demokratische Gestaltung der Lebensverhältnisse, während andere sie mit Praktiken der Aussonderung, mit Krankenmord, Eugenik und Rassismus in Verbindung bringen. Der Begriff taucht bei Vertretern der Alten Rechten ebenso auf wie in neueren linksradikalen Texten, ihn verwenden Kritiker des biotechnologischen Fortschritts, aber auch dessen Befürworter, erklärte Rassisten wie bekennende Marxisten.

1 Dieser Artikel beruht auf Argumenten, die systematisch in meinem Buch Biopolitik zur Einführung (Lemke 2007) entfaltet werden.

2 Vgl. etwa die Beiträge in einem kürzlich veröffentlichten Lexikon der Biopolitik (Brandimarte et al. 2006). 
Offenbar meint jeder etwas anderes, wenn von Biopolitik die Rede ist. Dabei scheint doch klar zu sein, was der Begriff bezeichnet. Dem Wortsinn nach meint Biopolitik die Politik, die sich mit dem Leben (griech.: bíos) befasst. Aber hier fangen die Probleme schon an. Denn was für die einen wie eine Banalität klingt („Befasst sich Politik nicht immer mit dem Leben?“), gilt anderen als Ausschlusskriterium: Politik beginne - so die hier vertretene Auffassung - erst dort, wo das biologische Leben ende, sie sei gemeinsames Handeln und Entscheiden, also eben das, was über das „,nur“ Kreatürliche und Körperliche hinausführt (vgl. Fehér/Heller 1995; Heller 1996). Auch über den zu veranschlagenden Zeitraum herrscht wenig Einigkeit. Reicht Biopolitik bis in die Antike, möglicherweise sogar bis zur Entstehung der Landwirtschaft zurück, ist sie Resultat biotechnologischer Neuerungen in der jüngeren Gegenwart oder bezeichnet sie gar - wie ein Kommentator meint - die „Schwelle eines neuen Zeitalters“ (Mietzsch 2002, 4)?

Ich will im Folgenden einen Systematisierungsvorschlag machen, der helfen soll, Klarheit in das begriffliche Wirrwarr zu bringen. Ausgangspunkt für das hier vorgeschlagene Analyseraster ist die polare Grundkonstellation, die in der Zusammenführung von Leben und Politik zum Begriff der Biopolitik prinzipiell angelegt ist. ${ }^{3}$ Die vorliegenden Konzepte unterscheiden sich danach, auf welchen Bestandteil des Wortes sie den Akzent legen. Entsprechend lassen sich naturalistische Konzepte, die das Leben als vorpolitische Grundlage der Politik begreifen, von politizistischen Ansätzen abgrenzen, die Lebensprozesse als außerpolitischen Gegenstand der Politik in den Blick nehmen. Die beiden Interpretationslinien werden in den ersten beiden Teilen des Beitrags vorgestellt. Meine Hauptthese ist, dass beide Ansätze zentrale Dimensionen biopolitischer Prozesse nicht erfassen. Gegen die naturalistische und die politizistische Lesart soll im dritten Teil ein relationaler Begriff der Biopolitik vorgeschlagen werden, den als Erster der französische Philosoph und Historiker Michel Foucault entwickelt hat. Die in der Rezeption des foucaultschen Begriffs der Biopolitik formulierten Korrekturvorschläge und die theoretischen Weiterentwicklungen lassen sich in einer Analytik der Biopolitik synthetisieren, die ich im vierten Teil meines Beitrags präsentieren und gegen bioethische Reflexionsund Analyseformen profilieren werde.

\section{Leben als Grundlage der Politik: Organizistische, rassistische und biologistische Konzepte}

Die vermeintliche Naturbasis der Politik steht im Mittelpunkt eines heterogenen Ensembles von Theorien, das von den organizistischen Staatskonzepten des beginnenden 20. Jahrhunderts über rassistische Argumentationsmuster im Nationalsozialismus und in der Alten und Neuen Rechten bis hin zu biologistischen Ansätzen in der zeitgenössischen Politikwissenschaft reicht. Ich gehe nur auf einige wichtige Stationen ein, die hier schlaglichtartig beleuchtet werden.

Der schwedische Politikwissenschaftler Rudolf Kjellén dürfte einer der Ersten gewesen sein, der den Begriff der Biopolitik verwendete. ${ }^{4}$ Kjellén, bis zu seinem Tod 1922 Professor an der Universität Uppsala, vertrat eine organizistische Konzeption des Staates. Er begriff den Staat als Kollektivsubjekt, das über einen eigenen Körper und Geist verfügt und sah in Politik, Wirtschaft, Kultur und Recht lediglich verschiedene Ausprägun-

3 Für anders ansetzende Systematisierungsvorschläge vgl. Heins/Flitner 1998; Buchstein/Beier 2004.

4 Eine knappe Skizze zur Begriffsgeschichte findet sich in Esposito 2004, 6-16. Die Wortverbindung von „Leben“ und „Politik“ ist freilich noch älter, sie geht vermutlich bis in die zweite Hälfte des 19. Jahrhunderts zurück. 
gen derselben organischen Kräfte, die den Staat konstituieren und seine Eigenart bestimmen. Der Nationalstaat galt ihm als die natürliche Form des Staates, der dessen ,ethnische Individualität“ zum Ausdruck bringe $(1924,103)$. Der „Staat als Lebensform“ sei nicht zuletzt durch die gesellschaftlichen Kämpfe um Interessen und Ideen gekennzeichnet, als deren Träger er Klassen und Gruppen ausmachte.

„Angesichts dieser das Leben selbst kennzeichnenden Spannung [...] ist bei mir die Neigung erwacht diese Disziplin nach der besonderen Wissenschaft des Lebens, der Biologie, Biopolitik zu taufen; [...] Im Bürgerkrieg der sozialen Gruppen erkennt man nur allzu deutlich die Rücksichtslosigkeit des Lebenskampfes um Dasein und Wachstum wieder, während man zugleich innerhalb der Gruppen ein kräftiges Zusammenarbeiten für das Dasein feststellen kann.“ (1920, 93f.)

Kjellén stand nicht allein mit seiner Idee, den Staat als „lebendigen Organismus“ oder als „Lebewesen“ aufzufassen. Viele zeitgenössische Politikwissenschaftler und Staatsrechtler, aber auch Biologen und Mediziner begriffen den Staat als eine ursprüngliche Lebensform, die den Individuen und Kollektiven vorausgeht und den Rahmen ihres Handelns vorgibt (vgl. Uexküll 1920; Hertwig 1922; Roberts 1938). Alle sozialen und politisch-rechtlichen Bindungen beruhen - so die Grundannahme - auf einer gewachsenen Ganzheit, die das Echte und Ewige, das Gesunde und Wertvolle verkörpert. Der Bezug auf das „Leben“ dient hier zugleich als mythischer Ausgangspunkt wie als normative Richtschnur und ist jeder rationalen Begründung oder demokratischen Zwecksetzung entzogen.

Die antidemokratisch-konservative Stoßrichtung der organizistischen Staatskonzeption erfuhr in der Zeit der nationalsozialistischen Herrschaft schnell eine rassistische Färbung. Die weit verbreitete Metapher des „Volkskörpers“ bezeichnete nun eine autoritär geführte, hierarchisch strukturierte und rassisch homogene Gemeinschaft. Zentral für die nationalsozialistische Staats- und Gesellschaftskonzeption war erstens die Vorstellung, dass nicht Individuen, Gruppen oder Klassen, sondern in sich geschlossene Abstammungsgemeinschaften Subjekte der Geschichte seien. Diese Idee wurde ergänzt durch die Annahme einer natürlichen Hierarchie von Völkern und Rassen gemäß ihrer unterschiedlichen „erbbiologischen Güte“, so dass es nicht nur gerechtfertigt, sondern zwingend notwendig erschien, Individuen und Kollektive ungleich zu behandeln. Zweitens beruhte die nationalsozialistische Ideologie auf dem Glauben, dass sich soziale Verhältnisse und politische Probleme letztlich auf biologische Ursachen zurückführen lassen. ${ }^{5}$

Die Grundprinzipien der nationalsozialistischen Gesellschaftslehre erläuterte der Präsident des Reichsgesundheitsamtes, Hans Reiter, in einem Vortrag mit dem Titel „Unsere Biopolitik und das Auslandsdeutschtum“ aus dem Jahr 1934. Die Erkenntnis, dass die Vergangenheit, Gegenwart und die Zukunft jeder Nation durch „erbbiologische“ Gegebenheiten bestimmt sei, bilde die Grundlage einer ,neuartigen Gedankenwelt, die [so Reiter] über die politische Idee hinaus sich zu einer bisher unbekannten Weltanschauung“ $(1939,38)$ entwickelt habe. Die Folge dieser Einsicht sei ein neuer, biologisch begründeter Begriff von Volk und Staat: „Zwangsläufig wirkt dieser Gedankengang dahin, das biologische Denken als Grundlinie, Richtung und Unterbau

5 Für die Formulierung und Ausarbeitung ihrer Gesellschafts- und Staatskonzeption nutzte die nationalsozialistische Bewegung eine Vielzahl unterschiedlicher Quellen und rezipierte sozialdarwinistische ebenso wie pangermanistische und völkische Ideologien. Sie griff anthropologische, biologische und medizinische Konzepte und Ideen auf und wirkte umgekehrt auf die Theoriebildung und empirische Arbeit in diesen Disziplinen zurück. Da in den Texten der Nationalsozialisten häufig heterogene Elemente und theoretische Versatzstücke unvermittelt nebeneinander stehen, ist es schwer, von einer einheitlichen biopolitischen Konzeption zu sprechen (vgl. Weingart et al. 1992). 
jeder wirklichen Politik anzuerkennen.“ (ebd.) Das Ziel dieser Politik bestand darin, die „Lebenstüchtigkeit“ und die Leistung des deutschen Volkes zu heben. Konkret richteten sich die Anstrengungen auf die quantitative Vermehrung der Bevölkerungszahl und die qualitative Verbesserung der „Erbgüte“ des deutschen Volkes. Um Letzteres zu erreichen, schlägt Reiter Verfahren der positiven wie der negativen Eugenik vor. Zum einen sei „minderwertiger Nachwuchs“ zu vermeiden und zum anderen sollten all jene gefördert werden, die „biologisch wertvoll“" sind (ebd., 41).

Die nationalsozialistische Biopolitik umfasste jedoch mehr als „Auslese“ und „Ausmerze“. Die rassenpolitischen Gesetze, Verordnungen und Maßnahmen zielten nicht nur darauf, das Fortpflanzungsverhalten zu reglementieren und zu disziplinieren; sie enthielten auch Antworten auf die imaginierten Gefahren der „Rassenmischung“. Die Entwicklung und die Pflege des Erbmaterials war in dieser Perspektive nur möglich durch den Schutz vor dem „Eindringen fremden Blutes“ und die Bewahrung der „rassischen Eigenart“ (ebd., 39) des deutschen Volkes. Die Sorge um die Reinheit der eigenen „Rasse“ fällt zusammen mit dem Kampf gegen den inneren und äußeren Feind. An dieser Stelle verbinden sich biopolitische Ideen mit geopolitischen Überlegungen. Die Kombination der rassenpolitischen Programme mit der Doktrin vom „Lebensraum“ bot die ideologische Grundlage der imperialistischen Expansion des Nazi-Reichs (vgl. von Kohl 1933).

Auch nach dem Ende der nationalsozialistischen Herrschaft und den Gräueln des Zweiten Weltkriegs besitzt die rassistische Konzeption der Biopolitik eine erhebliche Anziehungskraft. Vertreter der Alten wie der Neuen Rechten nutzen bis heute den Begriff der Biopolitik, um die Ignoranz des „Zeitgeists“ gegenüber der „Rassenfrage“ zu beklagen und stellen die fortdauernde Relevanz der Kategorie der Rasse für die Gegenwart heraus. Wie bei den nationalsozialistischen Ideologen konzentriert sich die Krisendiagnose auf den Kampf zwischen unterschiedlichen Rassen und die Bedrohung durch „Rassenmischungen“ und „Degeneration“. Ein Beispiel dafür ist ein Buch von Jacques Mahieu, eines ehemaligen Mitglieds der Waffen-SS, der nach dem Krieg nach Argentinien flüchtete und dort als Politikwissenschaftler an verschiedenen Universitäten lehrte. Der Autor will in die „Grundlagen der Biopolitik“ einführen und sieht deren „wichtige Rolle“ heute darin, die Ursachen für die angeblich zunehmenden „Rassenkämpfe“ und „ethnischen Zusammenstöße" herauszuarbeiten (Mahieu 2003, 13). Über die Problemanalyse hinaus steht der biopolitische Dreiklang von Volk-Nation-Rasse im Buchtitel auch für Lösungswege aus der Krise. „Sinn der Biopolitik“ sei es, „die Gesamtheit der Erbvorgänge insofern in Rechnung zu stellen, als sie das Leben der menschlichen Gemeinschaften beeinflussen“ (ebd., 12; vgl. auch Nation Europa 1965). ${ }^{6}$

Neben der organizistischen und rassistischen Konzeption bildete sich Mitte der 1960er Jahre innerhalb der Politikwissenschaft eine weitere Variante des naturalistischen Ansatzes heraus. $\mathrm{Zu}$ diesem Zeitpunkt entstand ein neuer Theorieansatz, der schließlich international unter dem Namen „biopolitics“ bekannt wurde. Die „Biopolitologen“ (Somit/Peterson 1987, 108) nutzen biologische Konzepte und Forschungsmethoden, um Ursachen und Formen politischen Verhaltens zu untersuchen. In diesem heterogenen Forschungsfeld lassen sich grob vier Bereiche unterscheiden, denen die meisten Arbeiten zuzuordnen sind. Die Rezeption der neodarwinistischen Evolutionstheorie bildet den ersten Komplex. In dessen Zentrum steht die historisch-anthropologische Frage nach der

6 Für Analysen der biopolitischen Konzepte der Neuen Rechten vgl. Feit 1987, 93-120; Reinfeldt/Schwarz 1992. 
Entstehung des Menschen und den Ursprüngen von Staat und Gesellschaft. Eine zweite Gruppe von Forschungen greift ethnologische und soziobiologische Konzepte und Forschungsergebnisse auf, um sie für die Analyse politischen Verhaltens fruchtbar zu machen. Unter die dritte Kategorie fallen Arbeiten, die sich für physiologische Faktoren und deren möglichen Beitrag für die Erklärung politisch relevanter Handlungsformen interessieren. Ein vierter Schwerpunkt liegt auf praktisch-politischen Problemen (,biopolicies“), die sich aus Eingriffen des Menschen in seine eigene Natur und die Veränderungen der Umwelt ergeben (Flohr/Tönnesmann 1983, 18-27; Somit/Peterson 1987, 108; Kamps/Watts 1998, 17 f.; vgl. auch Somit/Peterson 1998, 561-569).

Gemeinsam ist allen Vertretern der „biopolitics“ die Kritik an der aus ihrer Sicht unzureichenden theoretischen und methodischen Orientierung der Sozialwissenschaften. Deren Selbstverständnis gründe sich - so die hier vertretene Auffassung - auf die irrtümliche Vorstellung vom Menschen als eines prinzipiell freien Wesens, messe Sozialisations- und Lernprozessen eine zu große Bedeutung zu und verkenne, dass menschliches (politisches) Verhalten zu einem wesentlichen Teil biologisch (mit-)bedingt sei. „Biopolitologen" gehen in der Regel nicht von einem linearen Determinationsverhältnis aus, sondern verweisen eher auf biologische „Wurzeln“ oder „Faktoren“, die Handlungsmotive und -spielräume politischer Akteure entscheidend (mit-)bestimmen sollen. Als theoretische Grundlage dient häufig die neodarwinistische Evolutionstheorie. Diese geht davon aus, dass sich im Verlauf der menschlichen Entwicklungsgeschichte eine Reihe von Verhaltensdispositionen herausgebildet haben, die menschliches Verhalten zwar nicht vollständig festlegen, es jedoch in einer Vielzahl von Lebensbereichen entscheidend prägen. Arbeiten im Umkreis der „biopolitics“ interessieren sich dabei vor allem für Konkurrenz und Kooperation, Bindung und Aggressivität, Dominanzverhalten und Hierarchiebildung. Diese Phänomene gingen letztlich - so die Annahme - auf evolutionsgeschichtliche Mechanismen zurück und führten zur Ausbildung von Emotionen, die Individuen tendenziell zu einem „biologischen günstigen“ Verhalten drängen (Flohr 1998, 28).

Gegen diese Forschungsperspektive sind eine Reihe wichtiger Einwände und Kritikpunkte formuliert worden (Saretzki 1990; Hettlage 1990; Strube 2001; Euchner 2001), von denen ich hier nur einen herausgreifen kann. Ein entscheidendes Defizit des biopolitics-Ansatzes ist, dass Vertreter dieser Forschungsrichtung symbolischen Aneignungsprozessen und kulturellen Deutungsmustern zu wenig Beachtung bei der Untersuchung politischer und sozialer Prozesse und Strukturen schenken. Damit kann nur ein sehr begrenzter Ausschnitt der Realität erfasst werden, da gesellschaftliche Phänomene allein unter dem Gesichtspunkt der Anpassung an natürliche Gegebenheiten verhandelt werden. Nicht in den Blick gerät, inwieweit umgekehrt die sozio-politische Evolution auf „,biologische Faktoren“ einwirkt und diese verändert. „Biopolitologen“ sehen folglich „den Menschen“ allein als Produkt biokultureller Entwicklungsprozesse, nicht jedoch auch als deren Produzenten.

Diese einseitige Perspektive blendet eine zentrale Dimension der gegenwärtigen Diskussion des Verhältnisses von Natur und Gesellschaft, Biologie und Politik aus: die Frage nach der institutionell-politischen Ausgestaltung und den gesellschaftlichen Antworten auf die „Naturproblematik“, die den Ausgangspunkt für die zweite Interpretationslinie von „Biopolitik“ bildet. 


\section{Leben als Gegenstand der Politik: Ökologische und technik- zentrierte Konzepte}

In den 1960er und frühen 1970er Jahren nahm eine andere Bedeutung von Biopolitik Gestalt an, die weniger auf die biologischen Grundlagen der Politik fokussierte, sondern Lebensprozesse als neuen Gegenstand politischen Denkens und Handelns entdeckte. Im Zeichen der ökologischen Krise, die zunehmend ins Bewusstsein von politischen Akteuren und sozialen Bewegungen trat, bezeichnete Biopolitik nun jene Steuerungs- und Regulierungsanstrengungen, die sich auf die Lösung und Bewältigung der weltweiten Umweltprobleme bezogen. Einen wichtigen Anstoß hierfür lieferte der Bericht des Club of Rome (Meadows et al. 1972), der auf der Grundlage von Modellierungsverfahren und Computersimulationen die ökologischen und demografischen Grenzen des ökonomischen Wachstums aufzeigte und politische Interventionen forderte, um den Prozess der Zerstörung natürlicher Lebensgrundlagen auf der Erde aufzuhalten.

In dieser Konstellation gewinnt der Begriff der Biopolitik ein neues Profil. Er steht für die Herausbildung eines neuen Politik- und Handlungsfeldes, das auf den Erhalt der natürlichen Lebensgrundlagen des Menschen zielt (Bruns 1977). Dies zeigt sich deutlich etwa in der sechsbändigen Buchreihe Politik zwischen Macht und Recht des Politikwissenschaftlers Dietrich Gunst, in der neben der Deutschland-, Verfassungs- und Außenpolitik auch der Biopolitik ein eigener Band gewidmet ist. Dem Autor zufolge umfasst sie „alle Bereiche, die sich mit Gesundheits- und Bevölkerungspolitik, mit Umweltschutz und Fragen der Zukunft der Menschheit befassen. Dieser Bereich der Politik ist in einer umfassenden Form verhältnismäßig neu und berücksichtigt den Umstand, daß Fragen des Lebens und des Überlebens in ihrer Bedeutung ständig wachsen.“ (Gunst 1978, 9) Die einzelnen Kapitel des 1978 erschienenen Buches befassen sich vor allem mit den politischen und sozialen Problemen, die aus der wachsenden Weltbevölkerung, der kritischen Ernährungslage und dem Hunger in vielen Ländern, der Verschmutzung der Luft und des Wassers, Rohstoffknappheiten und Mängeln in der Energieversorgung resultieren.

Die Vorstellung von Biopolitik als Sicherung und Schutz der natürlichen Lebensgrundlagen auf dem Planeten Erde wurde schon bald durch eine zweite Bedeutungskomponente ergänzt und schließlich überlagert. Die 1970er Jahre waren nicht nur das Jahrzehnt einer erstarkenden Umweltbewegung und einer wachsenden Sensibilisierung für ökologische Fragen, die Dekade zeichnete sich auch durch einige spektakuläre biotechnologische Innovationen aus. 1973 gelang erstmals die gentechnische Übertragung von DNA über Artgrenzen hinweg. Damit wurde es möglich, Erbinformationen verschiedener Organismen zu isolieren und neu zu kombinieren. Ebenso wurden in diesem Zeitraum pränataldiagnostische Untersuchungen zum Bestandteil der Schwangerenvorsorge und neue Reproduktionstechniken wie die künstliche Befruchtung entwickelt. Die wachsende Bedeutung der Gen- und Reproduktionstechnologien warf Fragen nach einer Regulierung und Steuerung des wissenschaftlichen Fortschritts auf. Zeigten die Forschungsergebnisse und die technologischen Anwendungen, wie kontingent und brüchig die Grenze zwischen Natur und Kultur ist, bedurfte es nun verstärkter politischer und rechtlicher Anstrengungen, um sie neu festzulegen. Zu regeln war, welche technologischen Verfahren unter welchen Bedingungen zulässig sind. Klärungsbedürftig war auch, welche Forschungsanstrengungen mit öffentlichen Mitteln gefördert und welche verboten werden sollen. Fragen wie diese führten schließlich zu einem zweiten - technikzentrierten Bedeutungskomplex von Biopolitik. Dieser tritt neben bioethische Erwägungen und Überlegungen und bezeichnet die kollektive Aushandlung und Verständigung darüber, ob das, was technologisch möglich ist, auch gesellschaftlich akzeptiert werden soll. Der Beg- 
riff bezeichnet in diesem Zusammenhang „die seit gut zwei Jahrzehnten anhaltende gesellschaftliche Thematisierung und Regulierung der Anwendung moderner Naturwissenschaft und Technik auf den Menschen“ (van den Daele 2005, 8; vgl. auch Geyer 2001).

In journalistischen Texten und politischen Erklärungen und Reden hat sich diese Bedeutung von Biopolitik in den letzten Jahren durchgesetzt. Der Begriff steht spätestens seit der Jahrtausendwende für administrative und rechtliche Regulierungsprozesse, die Grundlagen und Grenzen biotechnologischer Interventionen bestimmen - die 2002 gegründete und inzwischen wieder eingestellte Zeitschrift für Biopolitik enthält zahlreiche Illustrationsbeispiele dieser Interpretationsrichtung (vgl. Mietzsch 2002). Festhalten lässt sich also, dass „Leben“ seit den 1970er Jahren in zweierlei Hinsicht zum Referenzpunkt politischen Denkens und Handelns wurde. Zum einen setzt sich die Einsicht durch, dass die „Umwelt“ menschlicher Gesellschaften durch die etablierte Gesellschafts- und Wirtschaftsordnung bedroht ist und die Politik auf diese Herausforderung reagieren muss, um angemessene Antworten auf die ökologische Frage zu finden und die „Überlebensbedingungen der Menschheit" (Fetscher 1980) zu sichern. Zum anderen - und zur gleichen Zeit - wird aber durch die biowissenschaftlichen Entdeckungen und durch technologische Innovationen zunehmend prekär, worin genau die ,natürlichen Lebensgrundlagen“ bestehen und wie sich diese von „künstlichen“ oder „manipulierten“ Lebensformen unterscheiden.

Damit ist es möglich, eine ökologische Biopolitik, die konservative und defensive Ziele verfolgt und die Politik auf die Sicherung und den Erhalt der natürlichen Lebensgrundlagen verpflichtet, von einer technikbezogenen Lesart der Biopolitik zu unterscheiden, deren Vertreter eher an der dynamischen Entfaltung und produktivistischen Erweiterung von Lebensprozessen interessiert sind.

Einen umfassenden Begriff der Biopolitik, der beide Bedeutungskomplexe zusammenführt, schlägt der Berliner Philosoph Volker Gerhardt vor. Für ihn ist Biopolitik durch „drei Hauptaufgaben“ gekennzeichnet. Neben die „ökologische Sicherung der Lebensmöglichkeit“ und die „biologische Steigerung der Lebenserträge“ sei der medizinische Schutz der Lebensentfaltung getreten (Gerhardt 2002a, 45). Diese Komponente zeitgenössischer Biopolitik habe das Aufgabenfeld der Biopolitik stark verändert und erweitert. Es umfasse nun auch ,jene Fragen, in denen sich der Mensch zum Objekt seiner lebenswissenschaftlichen Erkenntnisse macht" (ebd., 44). Gerhardt beklagt eine breite Abwehrfront von Skeptikern und Mahnern, die von Kirchenvertretern bis hin zu Marxisten reiche, die „Biopolitik unter Generalverdacht“ (Gerhardt 2002b) stelle und irrationale Ängste vor den neuen Technologien schüre. Gerhardt fordert demgegenüber eine rationale Debatte über deren Chancen und Risiken und sieht die Politik in der Pflicht. Notwendig sei eine politische Verfassung/Kultur, die die Freiheitsrechte der Einzelnen respektiere und dafür Sorge trage, dass ,die Selbstzweckbindung des Menschen nicht preisgegeben wird" (Gerhardt 2002a, 44).

„Da die Biopolitik in besonderem Maß unseren Selbstbegriff als Menschen berührt, haben wir entschieden auf ihrer Bindung an die Grund- und Menschenrechte zu bestehen. Und da sie weitreichende Konsequenzen für unser individuelles Selbstverständnis haben kann, sind wir vor allem auch in unserer eigenen Lebensführung gefordert. Wer nicht will, dass die Biotechnologie sich in jene Fragen mischt, die unter dem diskreten Schutz der Liebe stehen, hat dies zunächst und vor allem selbst zu entscheiden.“ (ebd., 47) 
Der von Gerhardt vorgeschlagene Begriff der Biopolitik greift aus verschiedenen Gründen zu kurz. Auch hier kann ich nur auf einen Einwand genauer eingehen. ${ }^{7}$ Die entscheidende Frage, die weder bei Gerhardt noch bei anderen Vertretern eines politizistischen Begriffs der Biopolitik beantwortet wird, ist die Frage nach dem „Wir“, das regelmäßig als Adressat der Texte fungiert. Wer entscheidet über die Ausgestaltung der Biopolitik und befindet selbstbestimmt über die eigene Lebensführung? Die Fassung von Biopolitik als ein Teilbereich etablierter Politik greift insofern zu kurz, als sie unterstellt, dass die politische Sphäre in ihrer Substanz von den wachsenden technologischen Möglichkeiten der Steuerung von Lebensprozessen unberührt bleibt. Dies ist jedoch nicht der Fall. Die Brisanz biopolitischer Fragen rührt ja gerade daher, dass sie nicht nur Gegenstand politischer Willensbildung sind, sondern das politische Subjekt selbst erfassen. $\mathrm{Ob}$ etwa embryonale Stammzellen als Rechtssubjekte oder als Biomasse bewertet werden, ob neurobiologische Forschungen die Grenzen menschlicher Willensfreiheit aufzeigen oder nicht: In all diesen Fällen geht es nicht allein um die politische Bewertung von Technologien oder das Aushandeln eines politischen Kompromisses in einem Feld divergierender Interessen und Werthaltungen. Fraglich ist vielmehr vor allem, wer an solchen Entscheidungs- und Bewertungsprozessen teilnehmen soll und wie normative Konzepte individueller Freiheit und Verantwortung mit biologischen Faktoren zusammenwirken.

\section{Michel Foucaults relationaler Begriff der Biopolitik}

Im Folgenden möchte ich die These zur Diskussion zu stellen, dass beide Interpretationslinien entscheidende Dimensionen biopolitischer Prozesse nicht erfassen. Bei aller Unterschiedlichkeit teilen die politizistische und die naturalistische Position wichtige Grundannahmen. Sie postulieren eine stabile Hierarchie und ein äußerliches Verhältnis von Leben und Politik. Sehen die Vertreter des Naturalismus das Leben ,unterhalb“ der Politik, wobei es politisches Denken und Handeln anleiten und erklären soll, bestimmt die Gegenseite Politik „oberhalb“ des Lebens, sie sei mehr als „nur“ Biologie und gehe über die existenziellen Notwendigkeiten biologischer Existenz hinaus. Beide grundlegenden Perspektiven auf das Problem der Biopolitik halten jeweils einen Pol des Bedeutungsfeldes stabil, um von ihm ausgehend Variabilitäten in dem anderen zu erklären. Damit verfehlen sie jedoch die in der Konjunktur des Begriffs der Biopolitik virulent werdende Instabilität der Grenze zwischen „Leben“ und „Politik“ und die Einsicht in die Relationalität der scheinbar voneinander isolierten Pole. Der Begriff der Biopolitik signalisiert eine Art doppelte Negation (vgl. Nancy 2002): Anders als in der naturalistischen Position vorausgesetzt, stellt das Leben keine stabile ontologische und normative Referenz dar. Spätestens mit den biotechnologischen Innovationen zeigt sich, dass Lebensprozesse in einem Maße gestaltbar geworden sind, das jede Vorstellung einer vom menschlichen Handeln unberührten Natur überholt erscheinen lässt. In dieser Hinsicht kann Natur nur noch als integraler Bestandteil von Natur-Gesellschaftsverhältnissen begriffen werden. Andererseits wird aber auch immer deutlicher, dass Biopolitik für eine signifikante Veränderung des Politischen steht. Das Leben ist nicht nur Gegenstand politischen Handelns und tritt mit diesem in ein äußerliches Verhältnis, sondern affiziert den Kern des Politischen. Biopolitik ist weniger Ausdruck des Willens eines Souveräns, sondern zielt auf die Wohlfahrt aller, sie hat es eher mit Lebewesen als mit Rechtssubjekten zu tun - oder genauer: mit Rechtssubjekten, die zugleich Lebewesen sind.

7 Für eine ausführlichere Kritik vgl. Thomä 2002. 
Gegen die naturalistische und die politizistische Lesart soll hier ein relationaler Begriff der Biopolitik vorgeschlagen werden, den Michel Foucault in den 1970er Jahren entwickelt hat. „Biopolitik“ markiert bei Foucault den expliziten Bruch mit dem Versuch, politische Prozesse und Strukturen auf biologische Determinanten zurückzuführen. Statt von ursprünglichen und überzeitlichen Gesetzmäßigkeiten der Politik auszugehen, diagnostiziert Foucault eine historische Zäsur, eine Diskontinuität in der Praxis der Politik. In dieser Hinsicht bezeichnet Biopolitik eine spezifisch moderne Form der Machtausübung.

Foucaults Begriff der Biopolitik wendet sich aber nicht nur gegen die Vorstellung, Lebensprozesse als vorpolitische Grundlage der Politik zu betrachten; er hält auch kritische Distanz zu Theorieangeboten, die Lebensprozesse als außerpolitischen Gegenstand politischen Handelns betrachten. Foucault zufolge ergänzt Biopolitik nicht traditionelle politische Kompetenzen und Strukturmuster durch neue Gegenstandsbereiche und Sachfragen. Sie stellt keine Erweiterung der Politik dar, sondern verändert deren Kern, indem sie Konzepte politischer Souveränität reformuliert und sie neuen Formen politischen Wissens unterordnet. Biopolitik steht für eine Konstellation, in der die modernen Human- und Naturwissenschaften und die aus ihnen hervorgehenden Normalitätskonzepte das politische Handeln strukturieren und dessen Ziele bestimmen. Aus diesem Grund hat für Foucault Biopolitik weder etwas mit der ökologischen Krise oder einem veränderten Umweltbewusstsein zu tun, noch ist sie auf die Entstehung neuer Technologien zu reduzieren; vielmehr steht sie für eine fundamentale Veränderung in der Ordnung des Politischen: „den Eintritt des Lebens und seiner Mechanismen in den Bereich der bewußten Kalküle“ (Foucault 1977, 170). „Zum ersten Mal in der Geschichte reflektiert sich das Biologische im Politischen. [...] Aber die ,biologische Modernitätsschwelle' einer Gesellschaft liegt dort, wo es in ihren politischen Strategien um die Existenz der Gattung selber geht. Jahrtausende hindurch ist der Mensch das geblieben, was er für Aristoteles war: ein lebendiges Tier, das auch zu einer politischen Existenz fähig ist. Der moderne Mensch ist ein Tier, in dessen Politik sein Leben als Lebewesen auf dem Spiel steht." (ebd., $170 \mathrm{f}$.)

Foucaults Begriff der Biopolitik zeigt auf, dass die scheinbar stabile Grenze zwischen Natur und Politik, die sowohl naturalistische wie politizistische Ansätze voraussetzen müssen, weniger Ausgangspunkt als Effekt politischen Handelns ist. Wenn das Leben selbst zum Gegenstand der Politik wird, hat dies Folgen für die Grundlagen, Instrumente und Ziele politischen Handelns. Biopolitik bezeichnet bei Foucault zunächst eine politische Ökonomie des Lebens, deren Ziel weniger in der Abschöpfung von Gütern, Produkten und Diensten als in Wertschöpfung besteht und die das Leben verwaltet, sichert und bewirtschaftet. Die Geburt der Biopolitik - so der Titel der Vorlesung Foucaults von 1979 - ist eng verbunden mit dem Auftauchen liberaler Regierungsformen. Foucault begreift den Liberalismus nicht als eine ökonomische Theorie oder eine politische Ideologie, sondern als eine spezifische Kunst der Menschenführung, die sich an der Bevölkerung als einer neuen politischen Figur orientiert. Der Liberalismus führt eine Rationalität des Regierens ein, die weder mittelalterliche Herrschaftskonzepte noch die frühneuzeitliche Staatsräson kannten: die Vorstellung einer Naturalität der Gesellschaft, die sowohl die Grundlage wie die Grenze des Regierungshandelns bildet. Dieses Naturkonzept ist kein traditionaler Rest oder ein vormodernes Relikt, sondern markiert einen bedeutenden historischen Einschnitt in der Geschichte politischen Denkens (Foucault 2004).

Scheinbar paradox dient der liberale Rückgriff auf die naturale Metaphorik vor allem dazu, die Natur hinter sich zu lassen - oder genauer: eine bestimmte Vorstellung der Natur, die diese als ewig und unveränderlich oder heilig ansah. Für die Liberalen ist Natur kein autonomer Bereich, in den prinzipiell nicht eingegriffen werden darf, sondern etwas, 
das vom Regierungshandeln selbst abhängt; Natur ist kein materielles Substrat, auf das die Regierungspraktiken Anwendung finden, sondern ihr ständiges Korrelat. Es ist zwar richtig, dass staatlichen Interventionen insofern eine „natürliche“ Grenze gesetzt ist, als sie mit der Naturalität der gesellschaftlichen Phänomene rechnen müssen. Dennoch ist diese Grenze keine negative; es ist vielmehr gerade die Natürlichkeit der Bevölkerung, die eine Reihe bis dahin unbekannter Interventionsmöglichkeiten eröffnet, die nicht notwendigerweise die Form von direkten Verboten und Vorgaben annehmen: „laisser-faire“, „anspornen“ und „,anreizen“ werden wichtiger als „reglementieren“, „,verordnen“ und „herrschen“. 8

Der Begriff der Biopolitik in den Schriften Foucaults verweist auf die Entwicklung eines spezifischen politischen Wissens und neuer Disziplinen wie Statistik, Demografie, Epidemiologie und Biologie, die Lebensprozesse auf dem Niveau von Bevölkerungen analysieren, um Individuen und Kollektive mittels korrigierender, exkludierender, normalisierender, disziplinierender, therapierender oder optimierender Maßnahmen zu ,regieren“. ${ }^{9}$ Hier spielt der eigentümliche Subjekt-Objekt-Status der politischen Figur „Bevölkerung“ eine wichtige Rolle. Steht sie einerseits für eine von politischen Interventionen prinzipiell unabhängige, sich durch eine ihr eigene Dynamik und Selbststeuerungskompetenzen auszeichnende kollektive Realität, ist andererseits diese Autonomie keine absolute Grenze politischer Interventionen, sondern im Gegenteil deren privilegierte Referenz. Die Entdeckung einer „Natur“ der Bevölkerung (etwa Geburten- und Sterblichkeitsraten, Krankheitsraten etc.) ist die Bedingung der Möglichkeit ihrer gezielten Beeinflussung und Steuerung.

Das Auftauchen der Bevölkerung als einer neuen politischen Figur im 18. Jahrhundert ist nicht zu trennen von der Entstehung der modernen Biologie. Liberale Konzepte von Autonomie und Freiheit sind eng an biologische Begriffe von Selbsterhaltung und Selbstregulation gekoppelt, die sich gegen das bis dahin vorherrschende physikalischmechanistische Paradigma der Untersuchung von Körpern durchsetzen. Die Biologie, die als Wissenschaft vom Leben um 1800 entsteht, geht von einem grundlegenden Organisationsprinzip aus, das die sichtbaren Phänomene des Lebens eher zufällig und ohne vorgezeichneten Plan entstehen lässt. An die Stelle einer äußeren Ordnung, die den Plänen einer höheren Instanz jenseits des Lebens entspricht, tritt eine innere Organisation, wobei „Leben“ als abstraktes und dynamisches Prinzip fungiert, das allen Organismen gleichermaßen eigen sei (Foucault 1971).

Auf diese Weise wird es möglich, das „Leben“ von dessen substanzhaften Trägern abzulösen. Nicht die singuläre Existenz von Menschen, sondern deren biologische Eigenschaften, die auf der Ebene von Bevölkerungen erhoben werden, sind Gegenstand der Biopolitik. Erst durch diese Abstraktionsleistung lassen sich Normen definieren, Stan-

8 Foucault zufolge lassen sich die Probleme der Biopolitik ,nicht vom Rahmen politischer Rationalität trennen [...], innerhalb dessen sie aufgetreten sind und ihre Zuspitzung erfuhren. Insbesondere nicht vom ,Liberalismus', denn durch die Beziehung auf ihn haben sie die Gestalt einer Herausforderung angenommen. Wie kann dieses Phänomen der ,Population' mit seinen spezifischen Wirkungen und Problemen in einem System Berücksichtigung finden, das auf die Respektierung des Rechtssubjekts und der Entscheidungsfreiheit bedacht ist? In wessen Namen und gemäß welchen Regeln kann man sie führen?“ (Foucault 2004, 435)

9 Foucault verwendet den Begriff der Regierung in einem „,sehr weitgefassten Sinn“ (Foucault 2005, 900). Er greift dabei die Vielfalt von Bedeutungen auf, die der Begriff noch bis in das 18. Jahrhundert hinein besaß. Während „Regierung“ heute vor allem Formen politischer Steuerung oder die rechtlichadministrative Struktur staatlicher Instanzen bezeichnet, bezog sich der Begriff früher auf die verschiedenen Formen der „Führung von Menschen“ und umfasste Formen der Fremdführung ebenso wie Techniken des Sich-Selbst-Regierens (vgl. dazu auch Lemke 1997). 
dards festlegen und Durchschnittswerte ermitteln. „Leben“ wird so zu einer selbstständigen, objektivierbaren und messbaren Größe und zu einer kollektiven Realität, die von den konkreten Lebewesen und der Partikularität individueller Lebenserfahrung abgelöst werden kann.

Zusammenfassend lässt sich festhalten, dass der Begriff der Biopolitik bei Foucault den Zusammenschluss von scheinbar Unvereinbarem bezeichnet. Wenn Politik im klassischen Sinn das ist, was jenseits der existenziellen Notwendigkeiten beginnt, enthält „Biopolitik" eine reflexive Dimension. Sie macht gerade das zum innersten Kern der Politik, was deren äußere Grenze darstellte: den Körper und das Leben. So gesehen schließt Biopolitik das ausgeschlossene Andere der Politik wieder ein. Allerdings sind nun weder die Politik noch das Leben, was sie vor dem Auftauchen der Biopolitik waren. Das Leben hört auf, das immer wieder vorausgesetzte, aber selten explizierte Gegenstück der Politik zu sein; es wird von der Singularität konkreter Existenzen abgelöst und zu einem Abstraktum, zum Gegenstand wissenschaftlichen Wissens, administrativer Sorge und technischer Optimierung. Und die Politik? Auch die Politik verändert sich im Zeichen biopolitischer Rationalitäten und Technologien. Sie macht sich abhängig von Lebensprozessen, die sie nicht selbst regeln kann und deren Selbststeuerungskapazitäten sie respektieren muss. Aber eben dadurch gewinnt sie ungemein an Interventions- und Gestaltungsoptionen. Sie vervielfacht ihre Möglichkeiten, indem sie neben den direkten Formen autoritativer Steuerung nun über viele indirekte Mechanismen der Anreizung und Anleitung, der Vorsorge und Vorhersage, der Moralisierung und Normalisierung verfügt. Sie kann vorschreiben und verbieten, aber auch anregen und anreizen. Sie kann reglementieren und disziplinieren - oder eben aktivieren und autonomisieren. ${ }^{10}$

Der Foucaultsche Blick auf das Problem der Biopolitik erlaubt es, die naturalistischen und politizistischen Grundpositionen als konstitutive Bestandteile einer gemeinsamen biopolitischen Problematik sichtbar zu machen. Die Konzeption der Natur als interventionsfrei und schicksalhaft ist die Kehrseite ihrer zunehmenden wissenschaftlichen und technologischen Durchdringung (vgl. Latour 1995). Beide Perspektiven reduzieren gleichermaßen die Bedeutung der Politik, der etwas Reaktives, Abgeleitetes und Nachträgliches anhaftet. In der naturalistischen Version beschränkt sie sich darauf, die Ordnung der Natur zu reproduzieren; sie soll ausdrücken, was durch biologische Evolutionsprozesse vorherbestimmt sei. In der politizistischen Variante erscheint sie als bloßer Reflex wissenschaftlich-technologischer Prozesse, deren Anpassung an gesellschaftliche Verhältnisse sie regulieren und gestalten soll. Gegen diese beiden grundlegenden - zugleich einander entgegengesetzten wie komplementären - Positionen soll hier abschließend eine Analytik der Biopolitik skizziert werden, die die Bedeutung des Politischen ernst nimmt.

\section{Konturen einer Analytik der Biopolitik}

Eine Analytik der Biopolitik unterscheidet sich von naturalistischen und politizistischen Konzepten, da sie weder auf die Ursachen noch auf die Wirkungen der Politik des Lebens fokussiert, sondern deren Funktionsweise beschreibt. Nicht „warum“ oder „wozu“, sondern „wie“ steht in ihrem Zentrum. Ihr Problem ist weder die Biologisierung der Politik noch die Politisierung der Biologie, da sie „Leben“ und „Politik“ als Elemente

10 Allerdings hat Foucault diese Analyseperspektive eher angeregt als sie systematisch zu verfolgen. Er versäumt es, seine Ausführungen zum Verhältnis zwischen Biopolitik und Liberalismus zu konkretisieren, das eigentlich im Zentrum der Vorlesung von 1979 stehen sollte (vgl. 2004, 41-44; 116). Leider bleibt es bei der »Absicht « - wie Foucault im Verlauf der Vorlesung selbstkritisch einräumt (ebd., 260). 
eines dynamischen Beziehungsgeflechts statt als äußerliche und unabhängige Größen begreift.

Diese Untersuchungsperspektive hat ihren Ausgangspunkt in der von Michel Foucault eröffneten theoretischen Perspektive, sie „lebt“ aber vor allem von zahlreichen Korrekturen und Weiterentwicklungen. Zwei Rezeptionslinien lassen sich grob unterscheiden. Die Erste ist in Philosophie und Gesellschaftstheorie sowie in der allgemeinen Soziologie und der politischen Theorie beheimatet und konzentriert sich auf die Frage nach dem Modus des Politischen: Wie funktioniert Biopolitik und welche Gegenkräfte mobilisiert sie? Wie unterscheidet sie sich analytisch und historisch von anderen Epochen und Politikformen? Die zwei Extrempunkte dieser Diskussion stellen zugleich die prominentesten Beiträge in der Debatte dar: die Schriften von Giorgio Agamben auf der einen und die Arbeiten von Michael Hardt und Antonio Negri auf der anderen Seite (Agamben 2002; 2004; Hardt/Negri 2002; 2004). Die zweite Rezeptionslinie greift auf Forschungsergebnisse und Einsichten der Wissenschafts- und Techniksoziologie, der Wissenschaftsgeschichte, der Medizinsoziologie, der Kulturanthropologie, aber auch der feministischen Theorie und der Geschlechterforschung zurück (vgl. etwa Haraway 1995; Iacub 2001; Fassin 2005, Rose 2007). Sie interessiert sich für die Substanz des Lebens. Wenn in der Folge der biowissenschaftlichen Innovationen der lebendige Körper heute weniger als organisches Substrat denn als molekulare Software begriffen wird, die gelesen und umgeschrieben werden kann, dann stellt sich die Frage der Biopolitik in veränderter Weise: Was ist die Bedeutung von Leben innerhalb dieser neuen politisch-epistemologischen Konstellation?

Insgesamt haben die verschiedenen Rezeptionslinien den foucaultschen Begriff der Biopolitik in mehrfacher Hinsicht weitergeführt und konkretisiert. Sie machen zunächst deutlich, dass aktuelle biopolitische Praktiken sich auf ein verändertes und erweitertes Wissen vom Körper und von biologischen Prozessen gründen. Dabei wird der Körper weniger als ein physisches Substrat oder eine anatomische Maschine denn als ein informationelles Netzwerk begriffen. Darüber hinaus erwies es sich als notwendig, die Analyse biopolitischer Mechanismen um die Untersuchung von Subjektivierungsformen zu ergänzen, um zu erfassen, wie die Regulation von Lebensprozessen auf individuelle und kollektive Akteure einwirkt und zur Ausbildung neuer Identitäten beiträgt. Kurzum: Der Begriff der Biopolitik wurde im Anschluss an Foucault um die Analyse von Wissensregimen und Subjektivierungsformen erweitert. Eine „Analytik der Biopolitik“ sollte es erlauben, das Beziehungsgeflecht zwischen Machtprozessen, Wissenspraktiken und Subjektivierungsformen in den Blick zu nehmen. Entsprechend lassen sich drei Dimensionen dieses Untersuchungsansatzes unterscheiden (vgl. auch Rabinow/Rose 2006, 197f.).

Erstens erfordert Biopolitik ein systematisches Wissen vom „Leben“ und von „Lebewesen“. Wissenssysteme stellen kognitive und normative Karten bereit, die biopolitische Räume erst eröffnen und Subjekte wie Objekte von Interventionen spezifizieren. Sie machen die Realität des Lebens begreifbar und kalkulierbar, so dass sie gestaltet und verändert werden kann. Zunächst ist es daher erforderlich, das Wahrheitsregime (und dessen Selektivität) zu erfassen, das den Hintergrund biopolitischer Praktiken bildet: Welches Wissen vom Körper und von Lebensprozessen gilt als besonders relevant und aussagekräftig (und welche alternativen Wirklichkeitsdeutungen werden demgegenüber abgewertet oder marginalisiert)? Welche wissenschaftlichen Experten und Disziplinen verfügen über legitime Autorität, die Wahrheit über das Leben, die Gesundheit, die Bevölkerung etc. zu sagen? In welchem Vokabular, in welchen Begriffen und Diskursen werden Lebensprozesse beschrieben, gemessen, kritisiert und bewertet? Welche kognitiven und intellektuellen Instrumente und welche technologischen Verfahren stehen für die Produk- 
tion von Wahrheit bereit? Welche Problemdefinitionen, welche Zielvorstellungen finden im Hinblick auf Lebensprozesse gesellschaftliche Anerkennung?

Das Problem des Wahrheitsregimes ist nicht von dem der Macht zu trennen. Es stellt sich also zweitens die Frage, wie Machtstrategien das Wissen vom Leben mobilisieren (und Machtprozesse Wissensformen hervorbringen und verbreiten). Auf diese Weise können Ungleichheitsstrukturen, Werthierarchien und Asymmetrien in den Blick genommen werden, die biopolitische Praktiken (re-)produzieren: Welche Lebensformen werden als sozial wertvoll, welche als „lebensunwert“ betrachtet? Welche existenziellen Notlagen, welche physischen und psychischen Leiden erhalten politische, medizinische, wissenschaftliche und soziale Aufmerksamkeit und werden als inakzeptabel, forschungsrelevant und therapiebedürftig begriffen - und welche werden vernachlässigt oder ignoriert? Wie schreiben sich Herrschaftsformen und Ausschlussmechanismen, Erfahrungen von Rassismus und Sexismus in die Körper ein und verändern diese (im Hinblick auf den Gesundheitszustand, die Lebenserwartung, das Erscheinungsbild etc.)? Ins Visier gerät auch die „Ökonomie“ der Politik des Lebens: Wer profitiert in welcher Form von der Regulierung und Optimierung von Lebensprozessen (finanzieller Gewinn, politischer Einfluss, wissenschaftliche Reputation, soziales Prestige etc.), wer trägt die Kosten und leidet darunter (Armut, Krankheit, vorzeitiger Tod etc.)? Welche Formen der Ausbeutung und Verwertung menschlichen und nicht-menschlichen Lebens lassen sich beobachten?

Drittens muss eine Analytik der Biopolitik auch die Subjektivierungsweisen in den Blick nehmen, die Art und Weise, wie Subjekte unter Anleitung wissenschaftlicher, medizinischer, moralischer, religiöser und anderer Autoritäten und auf der Grundlage gesellschaftlich wirksamer Körper- und Geschlechterordnungen, Gesundheits- und Krankheitskonzepte ihre eigene Existenz zum Gegenstand praktischer Bearbeitung machen. Auch hier ein Fragenkomplex, der wiederum nur einen Ausschnitt der relevanten Themen erfasst: Wie werden Menschen aufgerufen, sich im Namen des (individuellen und kollektiven) Lebens und der Gesundheit (der eigenen oder der Gesundheit der Familie, des Volkes, der Rasse etc.) im Hinblick auf definierte Ziele (Gesundheitsverbesserung, Lebensverlängerung, Erhöhung der Lebensqualität, Verbesserung des Genpools, Bevölkerungswachstum etc.) in bestimmter Weise zu verhalten (im Extremfall sogar für die Erreichung dieser Ziele zu sterben)? Wie werden sie dazu gebracht, ihr Leben als lebenswert bzw. lebensunwert zu erfahren? Wie werden sie als Angehörige einer „höheren“ oder „niederen“ Rasse, eines „starken“ oder „schwachen“ Geschlechts, eines ,aufstrebenden“ oder „degenerierten“ Volkes angerufen? Wie übernehmen und modifizieren Subjekte wissenschaftliche Deutungsmuster von „Leben“ für die eigene Lebensführung und begreifen sich etwa als von Genen gesteuerte Organismen, als neurobiologische Maschinen, als zusammengesetzte Körper, deren organische Bestandteile prinzipiell austauschbar sind? Wie lässt sich dieser Prozess als eine eigensinnig-aktive Aneignung und gerade nicht als rezeptiv-passive Annahme begreifen?

Was bringt ein solcher Ansatz für das Verständnis der Gegenwartsgesellschaften, worin besteht sein theoretischer „Mehrwert“ (vgl. dazu Fassin 2004, 178f.)? In historischer Hinsicht zeigt eine Analytik der Biopolitik, wie in den vergangenen Jahrhunderten nicht nur die Bedeutung von „Leben“ für die Politik zunahm, sondern sich auch die Definition des Politischen selbst veränderte. Vom reproduktiven Klonen über die Vogelgrippe bis zur Flüchtlingspolitik, von der Gesundheitsversorgung über die Rentenpolitik bis zum Bevölkerungsrückgang: Das individuelle und kollektive Leben, seine Verbesserung und Verlängerung, sein Schutz gegen alle Arten von Gefährdungen und Risiken nehmen heute einen immer größeren Raum in der politischen Debatte ein. Konnte sich der Wohlfahrtsstaat noch bis vor einiger Zeit auf die Probleme der Existenzsicherung konzentrieren, 
wird inzwischen auch die Regelung des Lebensanfangs und -endes an den Staat herangetragen. Damit wird auch die Frage virulent, wer zu welchem Zeitpunkt Mitglied der Rechtsgemeinschaft ist bzw. wer es noch nicht oder nicht mehr ist (Frage nach dem rechtlichen Status von Embryonen, Hirntoten etc.).

Empirisch erlaubt es eine Analytik der Biopolitik, Bereiche in Beziehung zu setzen, die meist durch administrative, disziplinäre und kognitive Grenzziehungen voneinander getrennt werden. Die kategorialen Unterscheidungen zwischen Natur- und Sozialwissenschaften, Körper und Geist, Natur und Kultur führen bei biopolitischen Fragen nicht weiter. Die Interaktionen von Leben und Politik können nicht allein mit sozialwissenschaftlichen Methoden und Forschungsansätzen bearbeitet werden. Ihre Untersuchung erfordert einen Disziplinen übergreifenden Dialog unterschiedlicher Wissenskulturen, Analysemodi und Erklärungskompetenzen. Ebenso ist es offenbar unzureichend, die medizinischen, politischen, sozialen und wissenschaftlichen Aspekte biopolitischer Probleme voneinander zu isolieren; die Herausforderung einer Analytik der Biopolitik besteht gerade darin, sie als Teil eines größeren Kontextes sichtbar zu machen - ein Kontext, der zahlreiche Grenzziehungen als empirische Sachverhalte enthält, die historisch erklärt und perspektivisch überwunden oder zumindest verschoben werden können.

Eine Analytik der Biopolitik erfüllt schließlich auch eine wichtige kritische Funktion. Sie zeigt, dass biopolitische Phänomene gerade nicht Resultat anthropologisch verankerter Triebe, evolutionsbiologischer Gesetze oder universeller politischer Zwänge sind, sondern nur im Rückgriff auf soziales Handeln und politische Entscheidungsprozesse begründet werden können. Diese Prozesse folgen keiner notwendigen Logik, sondern unterliegen einer spezifischen Rationalität, sie verkörpern institutionelle Präferenzen und normative Werthaltungen. Aufgabe einer Analytik der Biopolitik ist es, die ihnen eingeschriebenen Restriktionen und Kontingenzen, Zumutungen und Zwänge sichtbar und spürbar zu machen.

Das kritische Moment besteht hier nicht in der Zurückweisung des Bestehenden, sondern darin, Formen der Auseinandersetzung und Analyse zu entwickeln, die es erlauben, neue Möglichkeitshorizonte und Zielperspektiven wahrzunehmen - oder alte unter einem anderen Blickwinkel zu betrachten. Kritik besitzt in diesem Zusammenhang weniger eine negative oder destruktive als eine produktive und transformative Qualität. Sie basiert nicht auf dem universellen Geltungsanspruch wissenschaftlichen Wissens, eine letztgültige Definition der Wirklichkeit zu liefern, sondern rückt diesen Anspruch selbst in ein kritisches Licht, um dessen Partialität und Selektivität herauszustellen. Statt auf wissenschaftlich-autoritatives Wissen gründet sich eine Analytik der Biopolitik auf eine ethischpolitische Haltung: ein „Ethos“ oder „eine kritische Ontologie unserer selbst“ (Foucault 2005, 706). Dieses kritische Ethos erlaubt es, einen Weg jenseits der fruchtlosen Alternative der Trivialisierung und der Dramatisierung biopolitischer Phänomene zu skizzieren. Weder kann es überzeugen, Biopolitik zu entproblematisieren, um sie als bruchlose Verlängerung und Erweiterung Jahrtausende alter landwirtschaftlicher Produktions- und Züchtungsmethoden zu betrachten, wie Volker Gerhardt (2002a) dies tut; noch hat es umgekehrt Sinn, das Problem zu überzeichnen, um Vernichtungslager als Kulminationspunkt der Biopolitik zu beschreiben, wie dies in Giorgio Agambens Texten anklingt (vgl. Agamben 2002). In beiden Positionen treten allgemeine normative Präferenzen an die Stelle der zunächst erforderlichen empirischen Beschreibung.

Das kritische Ethos einer Analytik der Biopolitik könnte auch die derzeitige institutionelle und diskursive Dominanz der Bioethik aufbrechen. Diese hat entscheidend zu einer Beschränkung der öffentlichen Auseinandersetzung um das Verhältnis von Leben und 
Politik beigetragen, die vor allem in ethischen Begriffen und als Wertediskussion geführt wird (vgl. Gehring 2006, 8f.; Wehling 2007). Macht eine Analytik der Biopolitik die Komplexität eines Beziehungsgeflechts sichtbar, blendet der bioethische Diskurs umgekehrt die historische Genese und den sozialen Kontext biotechnologischer und biomedizinischer Innovationen regelmäßig zugunsten der Präsentation von Entscheidungsalternativen aus. Unberücksichtigt bleiben die epistemologischen und technologischen Grundlagen von Lebensprozessen sowie deren Einbindung in Machtstrategien und Subjektivierungsweisen. Der Akzent der Bioethik liegt auf abstrakten Entscheidungsoptionen - ohne zu prüfen, wer in welchem Umfang über materielle und intellektuelle Ressourcen verfügt, um tatsächlich von bestimmten technologisch-medizinischen Angeboten auch Gebrauch zu machen, und welchen gesellschaftlichen Zwängen und institutionellen Erwartungen die Einzelnen bei der Wahrnehmung der Optionen unterliegen.

Im Mittelpunkt der Bioethik steht die Frage: Was sollen wir tun? Sie reduziert Probleme auf behandel- und entscheidbare Alternativen, um Antworten auf „Nach-Fragen“ zu geben, während die Analytik der Biopolitik Probleme generiert und sich für die „VorFragen“ interessiert. Sie schärft den Blick für all jene historischen und systematischen Zusammenhänge, die regelmäßig außerhalb des bioethischen Rahmens und den Pro-oderContra-Alternativen verbleiben, sie eröffnet neue Fragehorizonte und Denkmöglichkeiten und überspringt etablierte disziplinäre und politische Grenzen. Eine Analytik der Biopolitik ist eine problematisierende und kreative Tätigkeit, sie verknüpft Gegenwartsdiagnostik und Zukunftsorientierung, indem sie scheinbar natürliche oder selbstverständliche Denk- und Handlungsmuster irritiert - und dazu einlädt, anders zu leben. Insofern besitzt eine Analytik der Biopolitik eine spekulative und experimentelle Dimension: Sie ist nicht die Affirmation dessen, was ist, sondern eine Antizipation dessen, was anders sein könnte.

\section{Bibliographie}

Agamben, G. (2002) Homo Sacer. Die souveräne Macht und das nackte Leben. Frankfurt/M.: Suhrkamp.

Agamben, G. (2004) Ausnahmezustand. Frankfurt/M.: Suhrkamp.

Brandimarte, R./Chiantera-Stutte, P./Di Vittorio, P./Marzocca, O./Romano, O./Russo, A./ Simone, A. (Hg.) (2006) Lessico di Biopolitica. Rom: Manifestolibri.

Bruns, H. (1977) Vorwort des Herausgebers. In: Gründler, H. (Hg.) Für Wahrheit und Redlichkeit im Lebensschutz und in der Biopolitik. Wiesbaden: Biologie-Verlag.

Buchstein, H./Beier, K. (2004) Biopolitik. In: Göhler, G./ Iser, M./Kerner, I. (Hg.) Politische Theorie. 22 umkämpfte Begriffe zur Einführung. Wiesbaden: VS Verlag.

Daele, W. van den (2005) Soziologische Aufklärung zur Biopolitik. In: ders. (Hg.) Biopolitik. Wiesbaden: VS Verlag.

Esposito, R. (2004) Bios. Biopolitica e filosofia. Turin: Einaudi.

Euchner, W. (2001) Politische Tiere - tierische Politik. Tradition und Wiederkehr der Zoologisierung des Politischen als biopolitics. In: Leviathan, 29: 371-410.

Fassin, D. (2004) Biopolitique. In: Lecourt, D. (Hg.) Dictionnaire de la Pensée Medicale. Paris: PUF

Fassin, D. (2005) Bio-Pouvoir ou bio-légitimité? Splendeurs et misères de la santé publique. In: Granjon, M.-C. (Hg.) Penser avec Michel Foucault. Théorie critique et pratiques politiques. Paris: Karthala.

Fehér, F./Heller, A. (1995) Biopolitik. Frankfurt/M., New York: Campus. 
Feit, M. (1987) Die „Neue Rechte“ in der Bundesrepublik. Organisation - Ideologie Strategie. Frankfurt/M., New York: Campus.

Flohr, H. (1998) Vom Wert der biologischen Verhaltensforschung für die Politische Soziologie. In: Konegen, N. (Hg.) Politik und Verwaltung nach der Jahrtausendwende Plädoyer für eine rationale Politik. Opladen: Leske und Budrich.

Flohr, H./Tönnesmann, W. (1983) Die Bedeutung der Life Sciences für die Politikwissenschaft. Selbstverständnis und Grundlagen von Biopolitics. In: dies. (Hg.) Politik und Biologie. Beiträge zur Life-Sciences-Orientierung der Sozialwissenschaften. Berlin, Hamburg: Paul Parey Verlag.

Foucault, M. (1971) Die Ordnung der Dinge. Frankfurt/M.: Suhrkamp.

Foucault, M. (1977) Der Wille zum Wissen. Sexualität und Wahrheit 1. Frankfurt/M.: Suhrkamp.

Foucault, M. (2004) Geschichte der Gouvernementalität II: Die Geburt der Biopolitik. Frankfurt/M.: Suhrkamp.

Foucault, M. (2005) Schriften. Band 4: 1980-1988. Frankfurt/M.: Suhrkamp.

Gehring, P. (2006) Was ist Biomacht? Vom zweifelhaften Mehrwert des Lebens. Frankfurt/M., New York: Campus.

Gerhardt, V. (2002a) Was Biopolitik ist und was gegen sie spricht. In: Zeitschrift für Biopolitik, 1 (1): 43-47.

Gerhardt, V. (2002b) Biopolitik unter Generalverdacht. In: Die Welt, 5. April 2002: 29.

Geyer, C. (Hg.) (2001) Biopolitik. Die Positionen. Frankfurt/M.: Suhrkamp.

Gunst, D. (1978) Biopolitik zwischen Macht und Recht. Mainz: von Hase und Köhler Verlag.

Haraway, D. (1995) Die Neuerfindung der Natur. Primaten, Cyborgs und Frauen. Frankfurt/M., New York: Campus.

Hardt, M./Negri, A. (2002) Empire. Die neue Weltordnung. Frankfurt/M., New York: Campus.

Hardt, M./Negri, A. (2004) Multitude. Krieg und Demokratie im Empire. Frankfurt/M., New York: Campus.

Heins, V./Flitner, M. (1998) Biologische Ressourcen und „Life Politic“. In: Flitner, M./Görg, C./Heins, V. (Hg.) Konfliktfeld Natur. Biologische Ressourcen und globale Politik. Opladen: Leske und Budrich.

Heller, A. (1996) Has Biopolitics Changed the Concept of the Political? Some Further Thoughts About Biopolitics. In: Heller, A./Puntscher Riekmann, S. (eds.) Biopolitics. The Politics of the Body, Race and Nature. Aldershot u.a.: Ashgate.

Hertwig, O. (1922) Der Staat als Organismus. Gedanken zur Entwicklung der Menschheit. Jena: Gustav Fischer Verlag.

Hettlage, R. (1990) Das Tier im Menschen - die verspätete Suche nach biologischen Ursachen. In: Göhler, G./Lenk, K./Schmalz-Bruns, R. (Hg.) Die Rationalität politischer Institutionen. Interdisziplinäre Perspektiven. Baden-Baden: Nomos.

Iacub, M. (2001) Les biotechnologies et le pouvoir sur la vie. In: Eribon, D. (Hg.) L'infréquentable Michel Foucault. Renouveaux de la pensée critique. Paris: EPEL.

Kamps, K./Watts, M. (Hg.) (1998) Biopolitics - Politikwissenschaft jenseits des Kulturalismus. Liber Amicorum Heiner Flohr. Baden-Baden: Nomos.

Kjellén, R. (1920) Grundriß zu einem System der Politik. Leipzig: S. Hirzel Verlag.

Kjellén, R. (1924) Der Staat als Lebensform. Berlin: Kurt Vowinckel Verlag.

Kohl, L. von (1933) Biopolitik und Geopolitik als Grundlagen einer Naturwissenschaft vom Staate. In: Zeitschrift für Geopolitik, 10: 305-310. 
Latour, B. (1995) Wir sind nie modern gewesen. Versuch einer symmetrischen Anthropologie. Berlin: Akademie.

Lemke, T. (1997) Eine Kritik der politischen Vernunft. Foucaults Analyse der modernen Gouvernementalität. Hamburg, Berlin: Argument.

Lemke, T. (2007) Biopolitik zur Einführung. Hamburg: Junius.

Mahieu, J. de (2003) Volk - Nation - Rasse. Grundlagen der Biopolitik. Riesa: Deutsche Stimme-Verlag.

Meadows, D. L./Meadows, D. H./Zahn, E./Milling, P. et al. (1972) Die Grenzen des Wachstums - Bericht des Club of Rome zur Lage der Menschheit. Stuttgart: DVA.

Mietzsch, A. (2002) Die Zeitschrift für Biopolitik - ein interdisziplinäres Medien-Projekt. In: Zeitschrift für Biopolitik, 1 (1): 3-4.

Nancy, J.-L. (2002) Note sur le terme „biopolitique“. In: ders. La création du monde ou la mondialisation. Paris: Gallilée.

Nation Europa (1965) Beiheft: Biopolitik. Coburg.

Rabinow, P./Rose, N. (2006) Biopower Today. In: Biosocieties, 1 (2): 195-217.

Reinfeldt, S./Schwarz, R. (1992) Biopolitische Konzepte der Neuen Rechten. In: Reinfeldt, S. (Hg.) Bio-Macht. Duisburg: DISS.

Reiter, H. (1939) Unsere Biopolitik und das Auslandsdeutschtum. In: ders. Das Reichsgesundheitsamt 1933-1939. Sechs Jahre nationalsozialistische Führung. Berlin: Julius Springer Verlag.

Roberts, M. (1938) Bio-Politics. An Essay on the Physiology, Pathology and Politics of the Social and Somatic Organism. London: Dent.

Rose, N. (2007) The Politics of Life Itself. Biomedicine, Power, and Subjectivity in the Twenty-First Century. Princeton, Oxford: Princeton University Press.

Saretzki, T. (1990) Biopolitics - ein erklärungskräftiger Ansatz für die Theorie politischer Institutionen? In: Göhler, G./Lenk, K./Schmalz-Bruns, R. (Hg.) Die Rationalität politischer Institutionen. Interdisziplinäre Perspektiven. Baden-Baden: Nomos.

Somit, A./Peterson, S. A. (1987) Introduction: Main Currents in Biopolitics In: International Political Science Review, 8 (2): 107-110.

Somit, A./Peterson, S. A. (1998) Review Article: Biopolitics After Three Decades - a Balance Sheet. In: British Journal of Political Science, 28: 559-571.

Strube, C. (2001) Zwei Kulturen der Rede von „Biopolitik“. In: Kleeberg, B./Metzger, S./Rapp, W./Walter, T. (Hg.) Die List der Gene. Strategeme eines neuen Menschen. Tübingen: Gunter Narr Verlag.

Thomä, D. (2002) Anmerkungen zur Biopolitik. Zwischen Gentechnologie und „Kampf der Kulturen“. In: Steiner, T. (Hg.) Genpool. Biopolitik und Körperutopien. Wien: Passagen Verlag.

Uexküll, J. von (1920) Staatsbiologie (Anatomie - Physiologie - Pathologie des Staates). Berlin: Verlag von Gebrüder Paetel.

Wehling, P. (2007) Biomedizische Optimierung des Körpers - individuelle Chance oder suggestive soziale Norm? In: Rehberg, K.-S. (Hg.) Die Natur der Gesellschaft. Verhandlungen des 33. Kongresses der Deutschen Gesellschaft für Soziologie in Kassel 2006. Frankfurt/M.: Campus (im Erscheinen).

Weingart, P./Kroll, J./Bayertz, K. (1992) Rasse, Blut und Gene. Geschichte der Eugenik und Rassenhygiene in Deutschland. Frankfurt/M.: Suhrkamp. 
Thomas Lemke ist Soziologe und Heisenberg-Stipendiat der Deutschen Forschungsgemeinschaft. Er arbeitet derzeit als wissenschaftlicher Mitarbeiter am Institut für Sozialforschung in Frankfurt am Main. Seine Forschungsschwerpunkte sind: Gesellschaftstheorie, politische Theorie, Gen- und Reproduktionstechnologien, Wissenschafts- und Techniksoziologie.

E-mail: lemke@em.uni-frankfurt.de 